\title{
Advances in Intelligent Assistive Technologies (IAT) for persons with dementia
}

\author{
Suzanne H. Hammad ${ }^{1}$, Dena Al-Thani ${ }^{2}$ \\ ${ }^{2}$ Hamad Bin Khalifa University
}

Advances in technologies and innovations over the past decade have sought solutions to ease the burden on caregivers and help persons with dementia live more independently and improve their overall wellbeing. Intelligent Assistive Technologies (IAT) for persons with ADRD encompass technologies that range from simple manual devices up to complex life monitoring and safety tracking GPS systems. This paper outlines recent developments in technology and innovation and their benefits. Generally defined as: "any device which assists a person in retaining or improving their independence, safety, security and dignity (Bonner \& Idris, 2012), such modern-day technologies are becoming more available in the market, and broadly include the following:

\section{Technologies for care management and activities of daily living}

Assistive devices can support older persons with dementia to schedule their medications in-take, swiftly connect with their loved ones, and much more. Special clocks have been designed to support ADRD patients' confusion associated with the differentiation between day and night hence reducing anxiety and fear symptoms that accompany the condition. Wearable tracking devices have long been used by dementia patients to protect them from the risk of being lost. Such devices are connected to an alert system that can help caregivers know the location of their loved ones. This technology can also be of important use in the case of personnel emergency. Smart home technologies such as Virtual Assistant such as Amazon Alexa and Google Assistant can be set to receive commands and questions from the users. These commands can include controlling the lighting, electricity plugs, heating and cooling systems, and security cameras. Everyday technologies can also be used to contact and assist people in managing their everyday lives, thus giving their caregivers respite. Technologies that are actively being explored include verbal live systems, digital television, and simple cell-phone interfaces that give individuals prompts to help them cope with activities of daily living. Live systems based on video monitoring are designed to track people engaged in specific tasks (e.g., making tea), to recognize if 
they get stuck at a particular point, and then give them a verbal prompt. (Dishman \& Carillo, 2016).

\section{Technologies for the physical and psychosocial wellbeing of the elderly}

Research in this area has been quite active in the past two decades resulting in many innovative ideas that tackle the day-to-day challenges faced by ADRD patients and their caregivers. In recent years, Ambient Assisted Living (AAL) technologies have been used as part of a range of services with the aim of improving the quality of life of older persons (e.g. Enshaeifar, 2018). This is achieved by deploying loT-enabled sensing devices within individuals' homes to capture and analyze the activity patterns of elderly people. These activity patterns are then combined with physiological measurements, including but not limited to heart rate, blood pressure, and body weight for the detection of cognitive changes. For example, researchers assess the health status of the patients by monitoring the electrocardiograph (ECG) activity and measuring the urinary volume using in-home sensors in the "Welfare Techno House" project (Tamura et al. 2007). Moreover, studies were done at the Centre for Advanced Studies in Adaptive Systems (CASAS) to make use of motion, door, and kinetic sensors to profile the occupant's everyday routine and focus on detecting drift in his/her everyday activities (Cook \& Krishnan, 2014). In the literature, researchers have studied motion density and movement patterns of the older population to discover and understand physical, cognitive, and perceptual decline (Rantz et al. 2011; Chernbumroong et al., 2014; Demiris et al., 2008). Since the condition of ADRD affects physical, cognitive, and behavioral aspects, in addition to emotional and social challenges such as anxiety, agitation, and stress, AT solutions must provide holistic and multi-level support to their users (lenca et. al, 2017). To that end, increasingly technologies that provide emotional and psychosocial support are being introduced or incorporated within existing devices. These seek to mitigate the effects of loneliness, enhance social connection, enhance mood, or reduce stress, alongside the other forms of assistance.

Given the immersive nature of Virtual Reality (VR) applications, several studies have examined their use to improve physical activities (de Vries et. al, 2018) reduce loneliness (Veldmeijer et. al, 2020), and support cognitive training (Garcia-Betances et. al, 2015). For instance, de Vries et al (2018) employed a VR system to promote regular exercise training through engaging the older person in a gamified immersive experience of skiing.

\section{Technologies to facilitate innovative delivery of health and social care}




\section{Nafath}

Issue 17 - May 2021

Another growing area of research and innovation is the development of social robots. However, patients with ADRD may express rejection when dealing with humanoid robotics, robots that look and feel like real human beings. They, therefore, generally prefer small robotics pieces with some animal traits such as robots that look and feel like pets. Telepresence robotic, a robotic-like AT that allow the older persons to connect with their family, friend, caregivers and support network through teleconferencing, has shown promising results in terms of the technology acceptance as they can ease the process of communication and hence facilitate the social interaction (Góngora Alonso et al., 2019). Robotic can also be used as a therapeutic tool to enhance physical activities. Such assistive systems could potentially prolong the safety and independence of older adults with ADRD, preventing accidents, assisting them during the completion of ADLs, facilitating caregiver supervision, and triggering the alarm in case of emergencies (Lotfi et al, 2012). Although they have been used long before Covid-19 pandemic, telecare solutions have also grown significantly as an outlet for care advice and monitoring from a distance.

\section{Conclusion}

There are many ways in which research and innovation in AT can help make a difference in the well-being of older persons allowing them to lead healthy and independent lives in their own environments, and a rapid review of the literature highlights their benefits and huge potential. And this is only the beginning. The challenge now is to ensure the accessibility, acceptability, and individualization of technologies to meet the needs of persons with ADRD across this progressive and complex disease.

\section{Reference}

Enshaeifar, S., Zoha, A., Markides, A., Skillman, S., Acton, S.T., Elsaleh, T., Hassanpour, M., Ahrabian, A., Kenny, M., Klein, S. and Rostill, H., (2018). Health management and pattern analysis of daily living activities of people with dementia using in-home sensors and machine learning techniques. PloS one, 13(5), p.e0195605.

Chernbumroong S., Cang S., Atkins A., and Yu H. (2014) Elderly activities recognition and classification for applications in assisted living, Expert Systems with Applications, vol. 40, no. 5, pp. 1662-1674, 2013.

Cook D. J. and Krishnan N., Mining the home environment. Journal of Intelligent Information Systems, vol. 43, no. 3, pp. 503-519.

Dale, 0. (2010) Usability and usefulness of GPS based localization technology used in dementia care. In Computers Helping People with Special Needs, Proceedings, Pt 1, Miesenberger K, Klaus J, Zagler W, Karshmer A, eds., pp.300-307. 
Nafath

Issue 17 - May 2021

Demiris, G., \& Hensel, B. K. (2008). Technologies for an aging society: a systematic review of "smart home" applications. Yearbook of Medical Informatics, 17(01), 33-40.

de Vries, A. W., Faber, G., Jonkers, I., Van Dieen, J. H., \& Verschueren, S. M. (2018). Virtual reality balance training for elderly: Similar skiing games elicit different challenges in balance training. Gait \& Posture, $59,111-116$.

Diaz-Orueta, U. and Konstantinidis, E. 2020. Shaping technologies for older adults with and without dementia: Reflections on ethics and preferences. Health Informatics Journal 2020, Vol. 26(4) 3215-3230

Dishman, E. and Carillo, M. C. (2007). Perspective on everyday technologies for Alzheimer's care: Research findings, directions, and challenges. Accessed 8 Feb 2021 at: https://www.alz.org/national/documents/etac_proceedings.pdf

Garcia-Betances, R. I., Jiménez-Mixco, V., Arredondo, M. T., \& Cabrera-Umpiérrez, M. F. (2015). Using virtual reality for cognitive training of the elderly. American Journal of Alzheimer's Disease \& Other Dementias $^{\circledR}, 30(1), 49-54$.

Góngora A., Hamrioui, S., de la Torre Díez, I., Motta Cruz, E., López-Coronado, M., \& Franco, M. (2019). Social robots for people with aging and dementia: A systematic review of literature. Telemedicine and EHealth, 25(7), 533-540.

lenca, M., Fabricea, O., Elgera, B., Caond, M., Pappagalloe, A.S., Kressigf, R. W., Wangmo, T.

(2017) Intelligent Assistive Technology for Alzheimer's Disease and Other Dementias: A Systematic Review. Available from:

https://www.researchgate.net/publication/313461148_Intelligent_Assistive_Technology_for_Alzheimer' s_Disease_and_Other_Dementias_A_Systematic_Review

Kieran, J., Egan, K. J. and Pot, A. M. (2016). Encouraging Innovation for Assistive Health Technologies in Dementia: Barriers, Enablers and Next Steps to Be Taken. JAMDA. 357-363. Available at:

https://www.jamda.com/action/showPdf?pii=S1525-8610\%2816\%2900048-7

Lotfi A, Langensiepen C, Mahmoud SM, Akhlaghinia MJ .(2012) Smart homes for the elderly dementia sufferers: Iden-tification and prediction of abnormal behaviour.J AmbientIntell Humaniz Comput3, 205218

Veldmeijer, L., Wartena, B., Terlouw, G., \& Veer, J. van't. (2020). Reframing loneliness through the design of a virtual reality reminiscence artefact for older adults. Design for Health, 4(3), 407-426.

https://doi.org/10.1080/24735132.2020.1848976 